\title{
Research on Resource Push Technology of Projectile Multi-dimensional Design
}

\author{
Xiao-Bing Gao, Rong Dong, Jun-Qiang Liu \\ School of Mechatronic Engineering \\ Xi'an Polytechnical University \\ Xi'an, China \\ E-mail: erbing@vip.sina.com
}

\begin{abstract}
In order to improve the level of the intelligent of product design, this paper designs resource push method based on projectile multi-dimensional design knowledge which can be edited and updated according to the characteristics of the fixture and tool part because KBE is the key technology in the process of product design and manufacturing, creates the reasoning process of product design based on smart fixture and tool library. The smart fixture and tool library becomes the configuration and reusable resources around product design.
\end{abstract}

Keywords-KBE; knowledge push; product model

\section{INTRODUCTION}

The fusion of product design process and knowledge[1, 2] has been a hot area of research of intelligent design and manufacture for several years. With the basic resource of product design process, fixture and tool resources play a very import role in the product of intelligent design. Fixture and tool resources are drived, build and selected by setting up process of product design knowledge representation for reuse recording, reusing and maintaining knowledge for engineer design. According to the pattern of " fixture and tool resources + knowledge $=$ intelligent resources", knowledge of application process is formed and participated in product design process based on tooling resources accumulating. The design process contains a lot of technical details and engineer design knowledge while there is no product design data in the whole process instead of related design result data. Fixture and tool resources as specific form and carrier of design process play important role in product design process.

The real difficulty is to complete multi-dimensional knowledge management of fixture and tool resources. (1)The product design process can be divided into multiple phases, but each design phase call for different design knowledge demand. It' $\mathrm{s}$ hard to push the right knowledge to every design phase. (2)There' $s$ a diversity of the data format being exchanged of fixture and tool resources. It ' $\mathrm{s}$ difficulty to design fixture and tool resources push method. Knowledge can be fully realized the combination of digital application and engineering practice with fixture and tool resources push method.

\section{MULTI-DIMENSIONAL DESIGN OF KNOWLEDGE PUSH DEPENDENT}

\section{A. The Structure of Knowledge Pushing}

Because of the need of projectile product development, The data form is established in the standard text editor. So the knowledge acquire method is established based on the repair knowledge model, which is shown as figure 1.

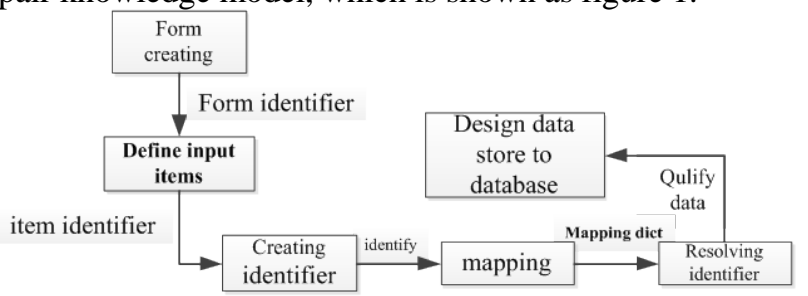

Figure 1. The knowledge acquire methods based on the knowledge model.

Assign unique form identification for each design form, denoted by TF, it is used as the unique name when identifying a form. Define the input items in the form and assign the unique data item identification for every input items, denoted by IF, which is used to identify the repair information when inputting the data. Create table structure of the corresponding form in the repair database at the same time, denoted by DT, create table field, denoted by FT. TF is corresponding to the input form in the database, Identification of the data item IF is corresponding to a repair data field FT. Define the identifier of every business data form, create a mapping relationship between the identifier, the input identification form TF and the identification of the data item IF, denoted by BM. A one-to-one mapping is created between each identifier $\mathrm{BM}$ and the unique identification of the data item from one form identification.

\section{B. The Structure of Knowledge Pushing}

Workflow is used to defined and specified design process because of projectile regular and fixed process[3]. The implementation of workflow summed up the role of the various design flow process, the condition and order of role, the role response of design process according to factory situation. The process-oriented workflow template is defined using formal description language. Workflow template is specified in creating new workflow by design process sponsor. Then knowledge is initiatively pushed by knowledge management platform without design process sponsor retrieval command. 
Based on the traditional workflow engine, knowledge of the workflow engine integrates design content and status. Its structure is shown in figure 2 .

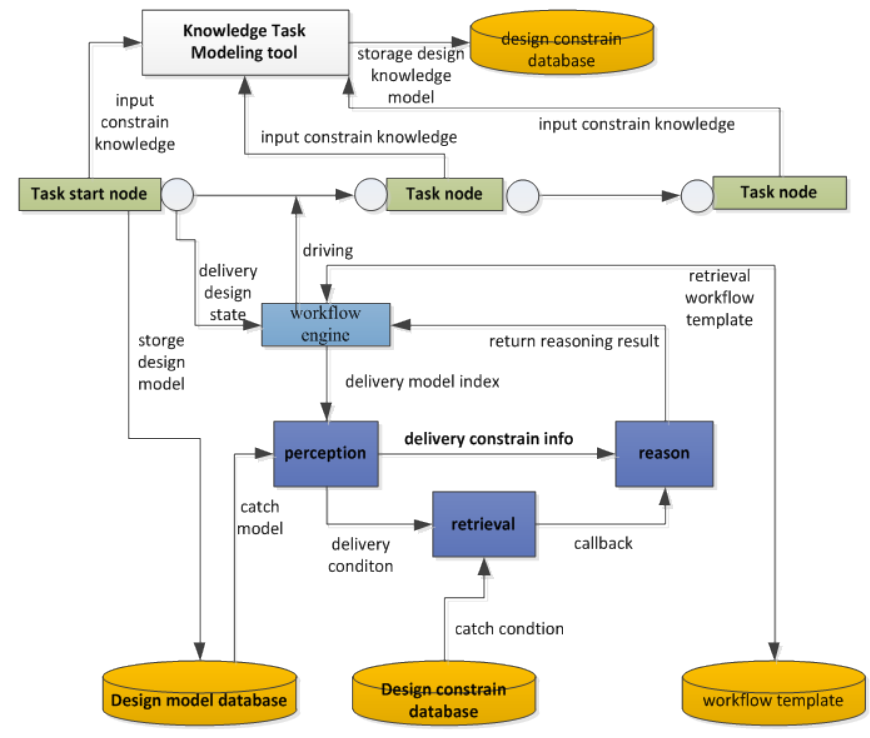

Figure2. The structure of knowledge pushing

\section{KNOWLEDGE PUSH ROLE OF FIXTURE AND TOOL RESOURCE}

\section{A. Push by Task Status of Process}

Each task state corresponds to the attributes of the content is: the design object parameters, the current design stage, the current job position, the current subject of professional. These properties have been stored in the background knowledge of the document or model mapping, so when using these attributes to retrieve knowledge, the current knowledge element space mission can be meet. The entire process is depicted in the Figure 3. The steps to push knowledge of fixture and tool resource are as follows:

- Step1: Capture the current design task attribute values of the designer with client of knowledge management platform.

- Step2: Call retrieval command to knowledge based on the condition of intersection of design task attribute.

- Step3: Get the knowledge document or list from the knowledge database.

- Step4: Access to knowledge document or model according to different evaluation criteria sorting attributes

- Step5: Sort the knowledge document or model according to the order property.

- Step6: Build links sorted documents or knowledge model, and package the link into knowledge unit.

- Step7: Pack knowledge package into the workflow engine.

- Step8: Send to the task of current working space automatically.
- Step9: Get the push reminds and catches the knowledge package in current working space.

- Step10: Open the package and get the knowledge detail.

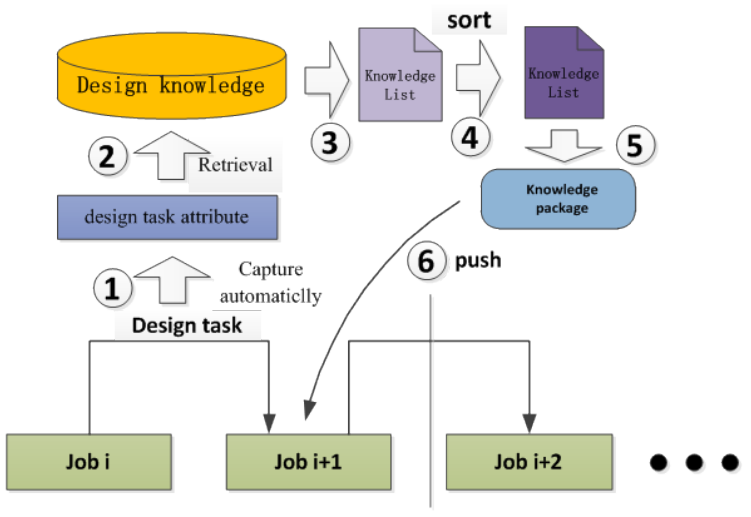

Figure 3. Push by task status of process.

\section{B. Push by Design Sponsor Role}

The design sponsor role can also determine the possible need for knowledge. The role is implied in the subject area which belongs to design sponsor, and it also represents sponsor's authority in the specify design. The entire process is depicted in the Figure 4.

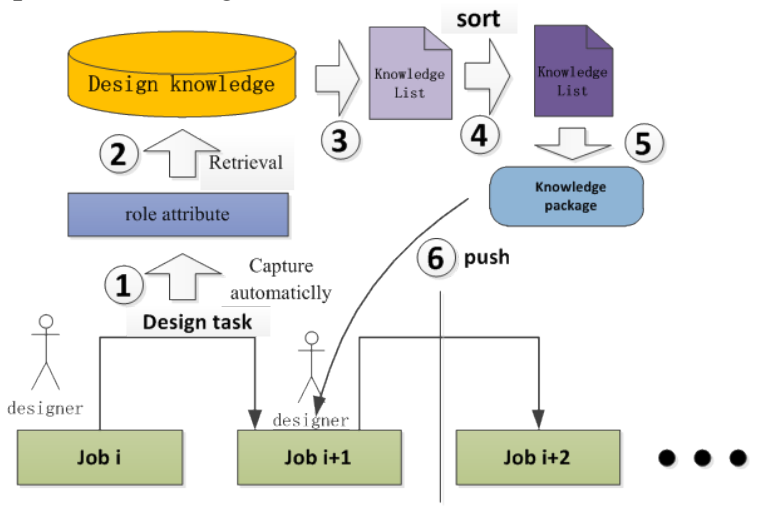

Figure 4. Push by design sponsor role.

The steps to push knowledge of fixture and tool resource are as follows.

- Step1: Capture the current role attribute values of the designer with client of knowledge management platform.

- Step2: Call retrieval command to knowledge based on the condition of intersection of role attribute.

- Step3: Get the knowledge document or list from the knowledge database.

- Step4: Access to knowledge document or model according to different evaluation criteria sorting attributes.

- Step5: Sort the knowledge document or model according to the order property.

- Step6: Build links sorted documents or knowledge model, and package the link into knowledge unit. 
- Step7: Pack knowledge package into the workflow engine.

- Step8: Send to the task of current working space automatically.

- Step9: Get the push reminds and catches the knowledge package in current working space.

- Step10: Open the package and get the knowledge detail.

\section{Push by Design Object and Command}

Knowledge requirement is hidden in design object and requirement. It can be reached by identifying the design object of the file type of the preliminary judgment of the design task type, determining product type through the typical characteristics of the CAD model and selecting design parameters in the design requirements of the parameter values. The entire process is depicted in the Figure 5.

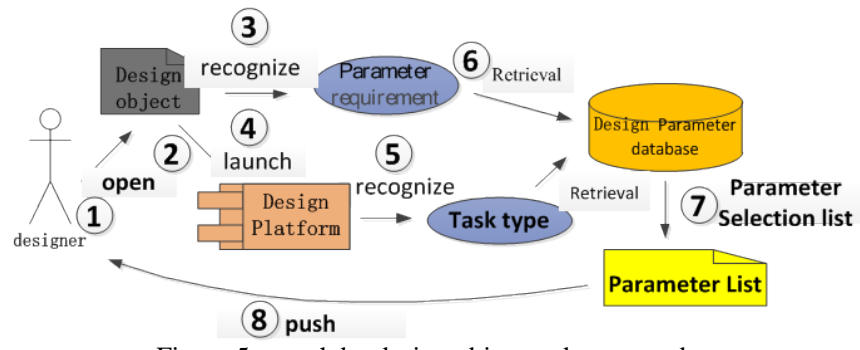

Figure 5. push by design object and command

The steps to push knowledge of fixture and tool resource are as follows.

- Step1: Identify design model type and open it with suitable design software while sponsor opening design model.

- Step2: Select the currently type design task for different types of documents.

- Step3: Catch the name and values of parameters from design task.

- Step4: Retrieval design parameter database with task type.

- Step5: Access to design parameters with the above of name and values of parameters.

- $\quad$ Step6: Build parameter list of selection range.

- Step7: Pack parameter list into the workflow engine, send the list to the current working space automatically.

\section{THE APPLICATION OF KNOWLEDGE PUSH OF FIXTURE AND TOOL RESOURCE}

\section{A. The Knowledge Learning Based on Markov Model}

The markov model[4, 5] is a kind of statistic model which is wildly used in various natural languages processing in application area. We can mine the knowledge of repair by training the using sequential patterns of repair data since MRO data can be seen as ordered repair data composition. In this paper, we assume that the subject of repair knowledge is $\mathrm{k}$, training markov model individually on the dataset of KLk In the markov model. Jump probability matrix Pand and initial probability vector are two essential parameters that need to be studied. Jump probability $\mathrm{P}\left(k_{i} \mid s_{i}^{k}\right)$ represents the given current data learning condition $S_{\bar{i}}$, the next data item that need to be studied is the probability of $K_{i}$. Use the jump probability matrix Pand, according to the present repair situation of the repair person, data items that are most likely to learn is predictable, establish the sequence of repair data items based on the context. The initial probability represents the probability that each maintenance data item is used as the initial data, the efficiency of knowledge learning is improved by recommending the higher popular initial probability item to the repair person. According to the maximum likelihood estimation, the calculation method for the jump probability is as in Eq. (1). that is the proportion of $\boldsymbol{K}_{\bar{i}}$ appears after $\boldsymbol{S}_{\bar{i}}$. Correspondingly, $K_{i}$ is the probability of the initial repair data item.

$$
P\left(k_{i} \mid S_{j}^{k}\right)=\text { frequency }\left(S_{j}^{k} \mid k_{i}\right) / \text { frequency }\left(S_{j}^{k}\right)
$$

In the markov model, with the growing of order $\mathrm{k}$, Add more context information to the model, the accuracy of prediction will go up correspondingly; but at the same time, data sparsity problem will reduce the coverage of forecast. Use the method of linear interpolation of first order model and second order model, strike a balance between accuracy and recall as in Eq. (2).

$$
P\left(k_{i} \mid S_{j}\right)=\lambda^{*} P\left(k_{i} \mid S_{j}^{1}\right)+(1-\lambda)^{*} P\left(k_{i} \mid S_{j}^{2}\right)
$$

$0 \leq \lambda \leq 1$ is the weight coefficient. Based on the markov model, the repair data can be organized as a sequence of repair knowledge that can be used in the maintenance process.

\section{B. Push by Design Object and Command}

The projectile design process knowledge is described as dynamic process knowledge[6], which can be defined, edited and reused. The knowledge push software system of projectile design based on workflow is shown in figure 6 .

The general process of projectile design is a kind of design process knowledge and design process. The new user selects some type of design process, in accordance with the specific design wizard can carry out fast, key links, the design process of key process in the design process in a clear expression and related design knowledge reasoning and pushing.

\section{CONCLUSIONS}

In order to further excavate internal potential, completes the optimizing process work, in the future work, need to be further from multiple channels to develop ideas for the realization of enterprise financial goals more active and make effort. The economic benefits of process optimization 
to produce are obvious. This paper described the process of knowledge push of fixture and tool resource in projectile multi-dimensional design, not only shorten the production period, improve the product quality, and reduce the projectile design cost.

\section{ACKNOWLEDGMENT}

The paper is supported by the China Postdoctoral Science Foundation, China (No.2014M552484) and the President Foundation of Xi'an Technological University, China (No.XAGDXJJ1304, No.XAGDXJJ1406)

\section{REFERENCES}

[1] Cope, B., M. Kalantzis and K.A. Mills, Doing Digital Composition on the Social Web: Knowledge Processes in Literacy Learning, B. Cope and M. Kalantzis, B. Cope and M. Kalantzis^Editors. 2015, Palgrave Macmillan UK. p. 172-185.
[2] Sunnersj, S., Problem Structure and Knowledge Processing. 2016, Springer International Publishing. p. 61-71.

[3] Chli, M. and P. de Wilde, Convergence and Knowledge Processing in Multi-Agent Systems. Advanced Information and Knowledge Processing. 2009: Springer London.

[4] Tveito, A. and G.T. Lines, Computing Characterizations of Drugs for Ion Channels and Receptors Using Markov Models. Lecture Notes in Computational Science and Engineering. 2016: Springer International Publishing.

[5] Buchholz, P., J. Kriege and I. Felko, Input Modeling with Phase-Type Distributions and Markov Models. SpringerBriefs in Mathematics. 2014: Springer International Publishing.

[6] Wolff, K.E., et al., Conceptual Knowledge Processing: Theory and Practice, K.E. Wolff, et al., K.E. Wolff, et al.^Editors. 2011, Springer Berlin Heidelberg. p. 1-25.

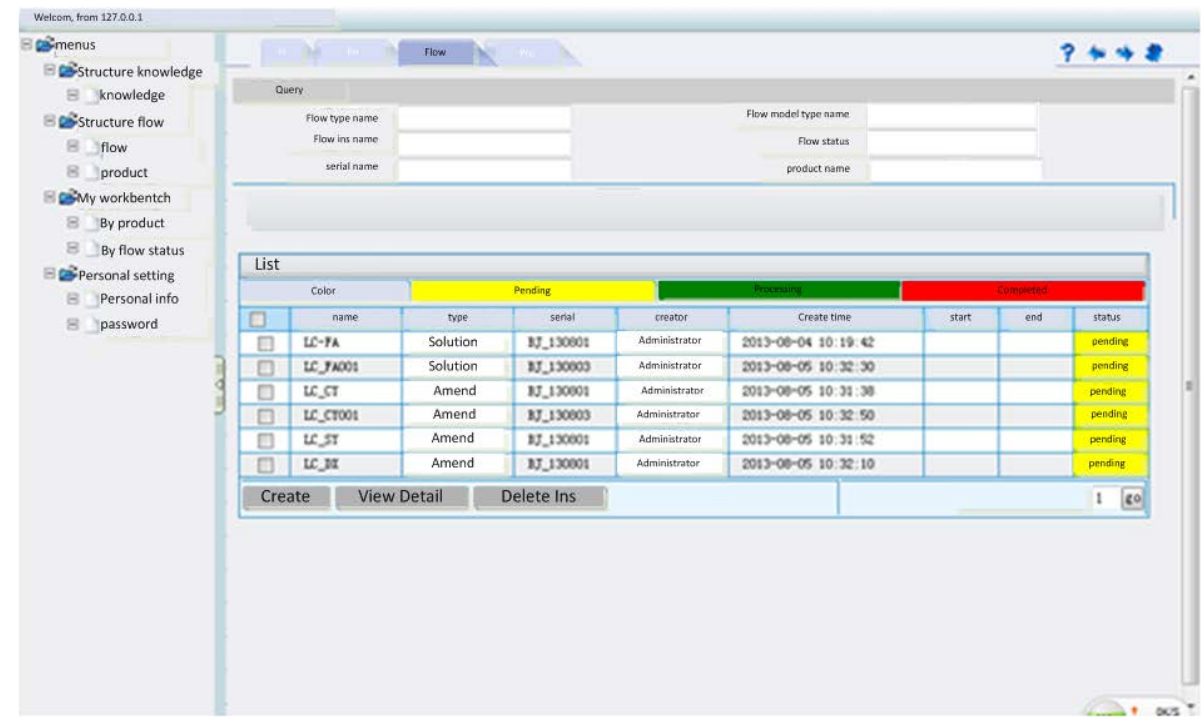

Figure 6. the knowledge push software system 